\title{
The convergence of three-dimensional printing and nail-art technology
}

\author{
Namsoo Peter Kim, $\mathrm{PhD}^{1,2} \mathbb{D}^{\mathbb{D}}$, Jihye Kim, $\mathrm{BS}^{1} \mathbb{1}$, Myung Sook Han, $\mathrm{PhD}^{3} \mathbb{D}$ \\ ${ }^{1}$ Center for Printable Materials Certificate, The University of Texas at El Paso, El Paso, TX, United States \\ ${ }^{2}$ Department of Metallurgical Materials and Biomedical Engineering, The University of Texas at El Paso, El Paso, TX, United States \\ ${ }^{3}$ Department of Beauty Design, Dongu College, Busan, Rep. of Korea
}

Background: With the convergence of internet of things (IoT) and artificial intelligence (Al) technologies with three-dimensional (3D) printing or additive manufacturing, the applications have extended to beauty arts and cosmetics.

Objective: The purpose of this research is to confirm the possibility of the convergence of IoT and Al 3D printing technology for cosmetics and beauty art for the 21 st century.

Methods: From January to October 2019, experienced 3D designers and customers were isolated, and more than 1,000 patterns were verified. Ultraviolet light-emitting diod (UV-LED) technology with built-in patented devices operating on a limited basis was used, and processes using low energies of 3 watts or less were applied. To control the 3D shape of the material, 3D printing technology with the z-axis at a height of 50 microns was applied to the shape to obtain a length of more than $3 \mathrm{~cm}$. Completed products realized via custom design and manufacturing processes were delivered to the customers who installed them on their own.

Results: More than 1,000 patterns were successfully printed through Wi-Fi from all over the world for Al-recommended nail art and tattoos. The stability of the loT has confirmed the quality of 3D printed nails and two-dimensional (2D) tattoos. Moreover, customers are not exposed harmful environments associated with tattoos and 3D nail art.

Conclusion: Application of 3D printing technology to the traditional nail art industry allows for the creation of sophisticated and customized shapes in a short period of time with a precision control of less than 50 microns in various colored 3D structures on artificial nail tips. This successful result of the convergence of $\mathrm{Al}$ and loT 3D printing technology in the field of nail art and tattoos is expected to apply further to the fields of cosmetology, makeup, plastic surgery, and art.

Keywords: artificial intelligence; convergence; cosmetics; internet of things; nail art; three-dimensional printing

\section{Introduction}

Nail art has grown to be the third pillar in the beauty industry, following hair care and skin care; currently, it is transforming into a cutting-edge industry surpassing simple color expression and beauty. As the social advancement of women has increased and economic living standards have improved, women have been applying various colors, patterns, and materials to express their identities through nail art. Accordingly, nail art has been recognized as a unique component in fashion, and the nail art industry has steadily expanded.

However, two main issues have emerged in the nail art industry that need to be addressed. The first is the unreliable reproducibility that occurs due to the reliance on handcrafted

Received December 9, 2019; Revised December 18, 2019; Accepted December 18, 2019

Corresponding author: Myung Sook Han

E-mail: mshan1009@hanmail.net

This is an Open Access article distributed under the terms of the Creative Commons Attribution Non-Commercial License (http://creativecommons.org/licenses/by-nc/4.0), which permits unrestricted non-commercial use, distribution, and reproduction in any medium, provided the original work is properly cited.

Copyright (c) 2019 Korean Society of Korean Cosmetic Surgery and Medicine (KSKCS \& KCCS). 
techniques, and the second is that consumers and providers are exposed to harmful substances throughout the nail art process. Competition in the industry is expected to increase due to the help of new scientific technologies that will decrease production time and reduce the cost of products. Meanwhile, improvement measures are in place to address the drastic increase in skin cancers, such as melanoma, dermal necrosis, and other skin diseases. Moreover, the scattering of acrylic materials during the polishing process of a manicure might cause fatal lung diseases [1]. In fact, stricter regulations concerning these issues have already been put into place in both New York and California in the United States [2].

Therefore, this study was strategically designed to implement a combined technology incorporating three-dimensional (3D) printing, the internet of things (IoT) system, and artificial intelligence (AI). This combined technology facilitates the creation of sophisticated colored 3D nail patterns while attempting to solve the aforementioned problems and further advance the future of the beauty industry.

\section{Materials and methods}

From January to October 2019, over 1,000 designs and their effectiveness were evaluated. An overview of the study is shown in Fig. 1. When a design file was selected by a client or recommended by AI, the file was uploaded remotely via Wi-Fi to the cloud. A Raspberry Pi (RPI) microprocessor recognized it and automatically uploaded the file to a Pull-down menu on the printer through a remote printer controlled by an IoT system. The artificial nails were then printed through an optimized $3 \mathrm{D}$ printing process that could print a two-dimensional (2D) shape. Furthermore, we used a technique that stably bonded a $3 \mathrm{D}$ printing result (using transparent ink) to the nails of a client without the need for chemical adhesives or a ultraviolet (UV) hardening process on-site.

For 3D nail printing, we selected and applied a process that minimized UV exposure and reduced energy usage to $3 \mathrm{~W}$ or less. This was done using a built-in UV light-emitting diode (UVLED) device that operated the curing on a limited basis while printing was completing. The technology selected for the $3 \mathrm{D}$ shape control applied the z-axis with a height of 50 microns $(\mu \mathrm{m})$ and built the shape to a length of $3 \mathrm{~cm}$ or higher.

\section{Results}

This unprecedented use of 3D IoT technology applied to nail art ensured environmental safety in that physical contact between the client and nail technician was not required. The test was performed approximately 1,000 times, and the technique was optimized such that a maximum of only 10 minutes was required for printing a set of ten nails. Through this process, it was confirmed that the time necessary to complete the printing was 30 minutes maximum from anywhere in the world (Fig. 2). As a result, the expression of details showed superior reproducibility and repeatability compared to the traditional human-handcrafted nail art. Because AI technology was used, clients received numerous recommendations for remarkable artificial nail art designs; this optimized the selection process and increased client satisfaction by its ability to meet many personal preferences. Nail designs could be revealed immediately in a final product. Furthermore, a logistics and supply method could be constructed to deliver the final products to consumers at a desired time and location, thereby allowing consumers to ultimately apply nail art in a remote location away from direct exposure to the chemical substances used in a traditional manicure. This study confirmed that cellphones, computers, and even printers could be connected, and the use of RPIs allowed consumers to find locations that facilitated easy deliveries and the shortest printing times possible; files could be sent in real time to a device possessed by a supplier through the network space.

\section{Discussion}

The precision, reproducibility, and repeatability of 3D print-

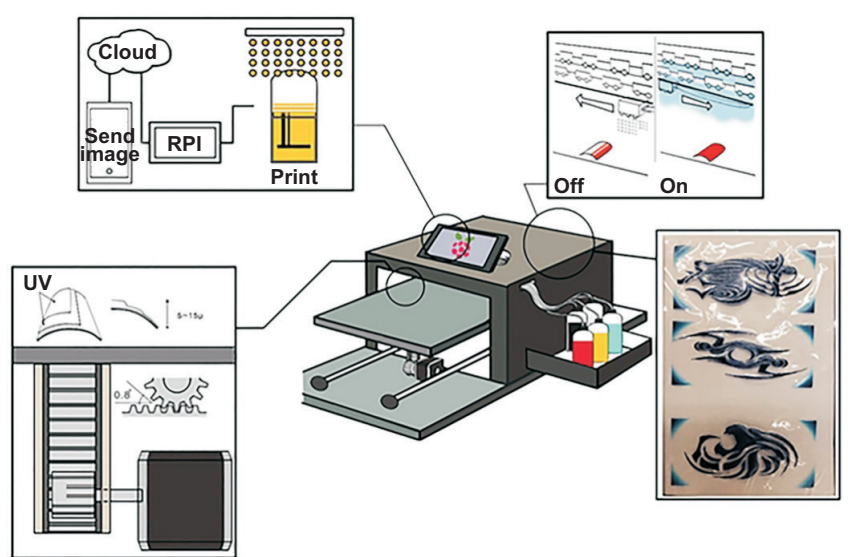

Fig. 1. Schematic diagram of three-dimensional nail art and tattoo printer, which uses a precision-controlled z-axis, internet of things (IoT) system, and artificial intelligence technology to reproduce designs recognized by the Raspberry Pi (RPI) microprocessor. UV, ultraviolet. 
ing is superior to traditional handcraft methods. Fig. 3 shows a unique design that was printed by recreating a classic $2 \mathrm{D}$ painting by Shin Yun-bok as a 3D image using the device tested during our study. Though the picture was reduced to a miniature size of $1 \mathrm{~cm} \times 1 \mathrm{~cm}$, its features remained clear and its 0.4 $\mathrm{cm}$ height was maintained, which confirmed the feasibility of $3 \mathrm{D}$ reproduction. In Fig. 3A, the print was created at the size of a credit card, and the decorative layer was formed in 3D. To apply this concept to nail art, the process and printing conditions were optimized over the course of 1,000 tests, and reproduceable 3D nail art was fabricated successfully in the shapes and sizes of fingernails. As seen in Fig. 3, the reduction and printing

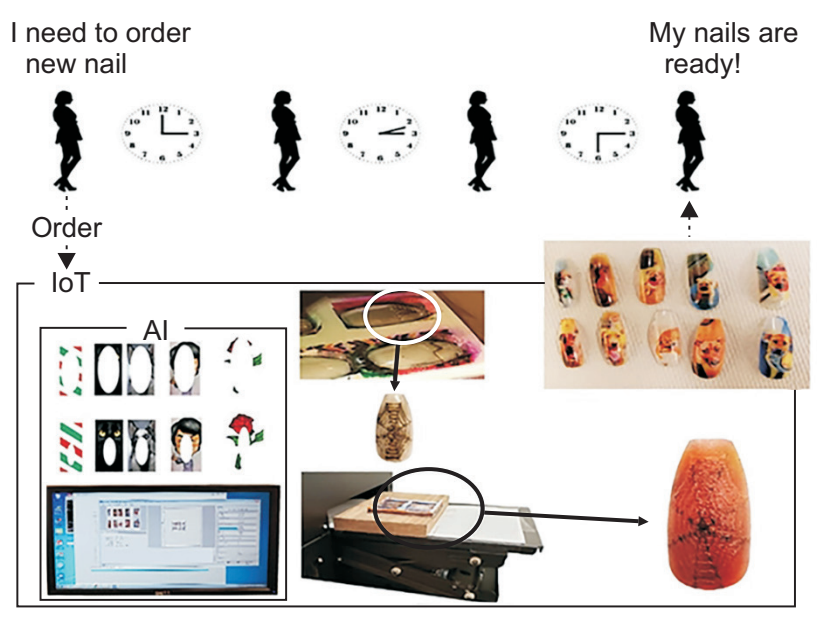

Fig. 2. Process of three-dimensional printing in real time with the internet of things (IoT) system and artificial intelligence (AI) technology. at a size of $1 \mathrm{~cm}^{2}$ shows the precision necessary to achieve the size of a fingernail. This was accomplished using a convergence of science and art that expressed the perspective in the painting by applying height differences between the decorative layers. The successful reproduction of a 3D shape using such materials was previously reported in a 2017 study, but an issue remained that prevented its commercialization, as it was unable to print on a curved surface, despite the appealing aspects achieved by the $3 \mathrm{D}$ shape.

This study documented an innovative attempt at printing high-definition, curved-surface patterns using a 2.5-W laser guide on the outer edges to increase the contact surface area and visibility on a curved surface using a Mysweety Mini Desktop Printer Engraver Machine (TwoWin, Shenzhen, China). The results indicated that this technology successfully increased the contact surface area and enhanced the precision of the material being applied, even on a curved surface.

Fig. 4A, C demonstrate how the laser guide applied nail tattoo, while Fig. 4B, D show that a high definition material of 4,000 dots per inch was precisely sprayed and cured on a curved 3D surface. In a rectangular frame, the artificial nails displayed more significant curvature than actual human fingernails. The curvature of the base of the artificial nails was adjusted according to the fingernails of each client, and as shown in Fig. 5 , a method of producing and outputting various shapes and colors was repeated successfully. Fig. 5A shows the results of the repeated ink-jetting method wherein $2.5 \mathrm{D}$ was applied to the materials. Fig. $5 \mathrm{~B}$ shows the perfect $3 \mathrm{D}$ shapes that were successfully reconstructed on curved surfaces by changing the

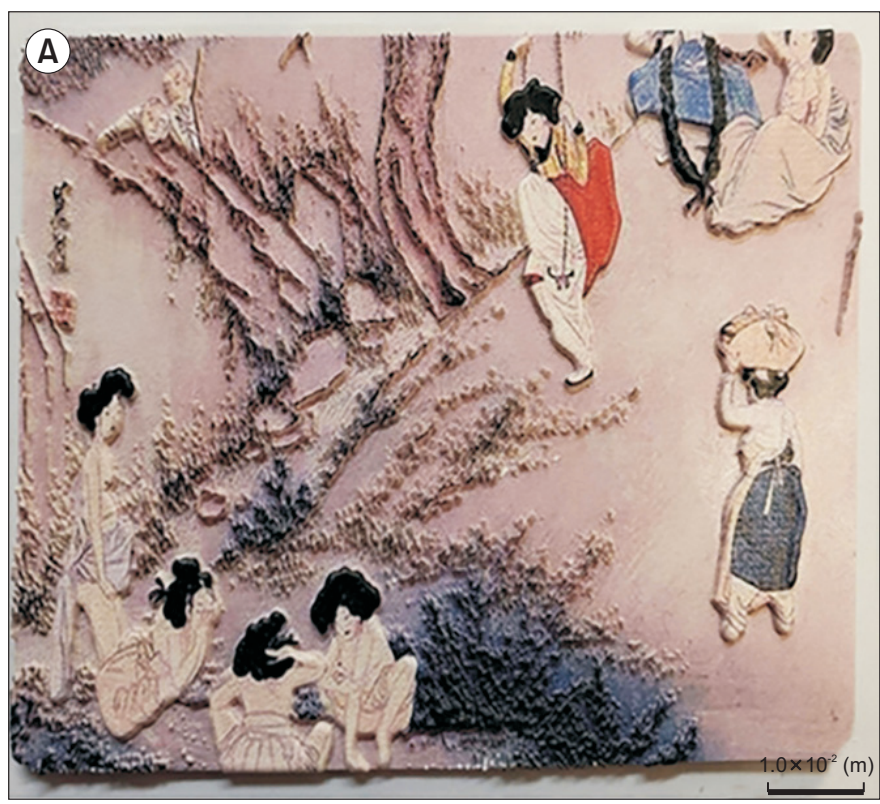

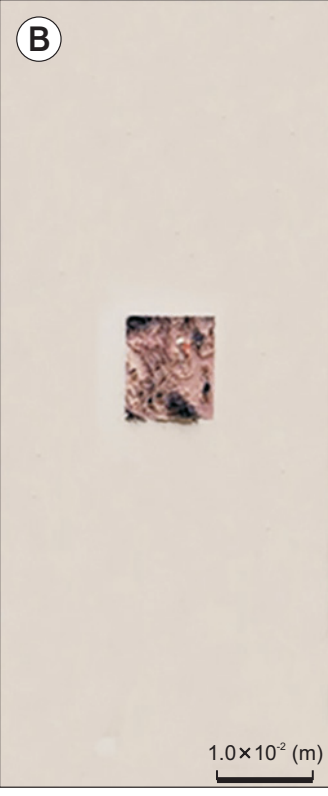

Fig. 3. Recreation of a classic painting by Shin Yun-bok from two-dimensional (2D) to three-dimensional (3D) in two different sizes: (A) credit card size and (B) $1 \mathrm{~cm}^{2} 3 \mathrm{D}$ structure for nail art. 


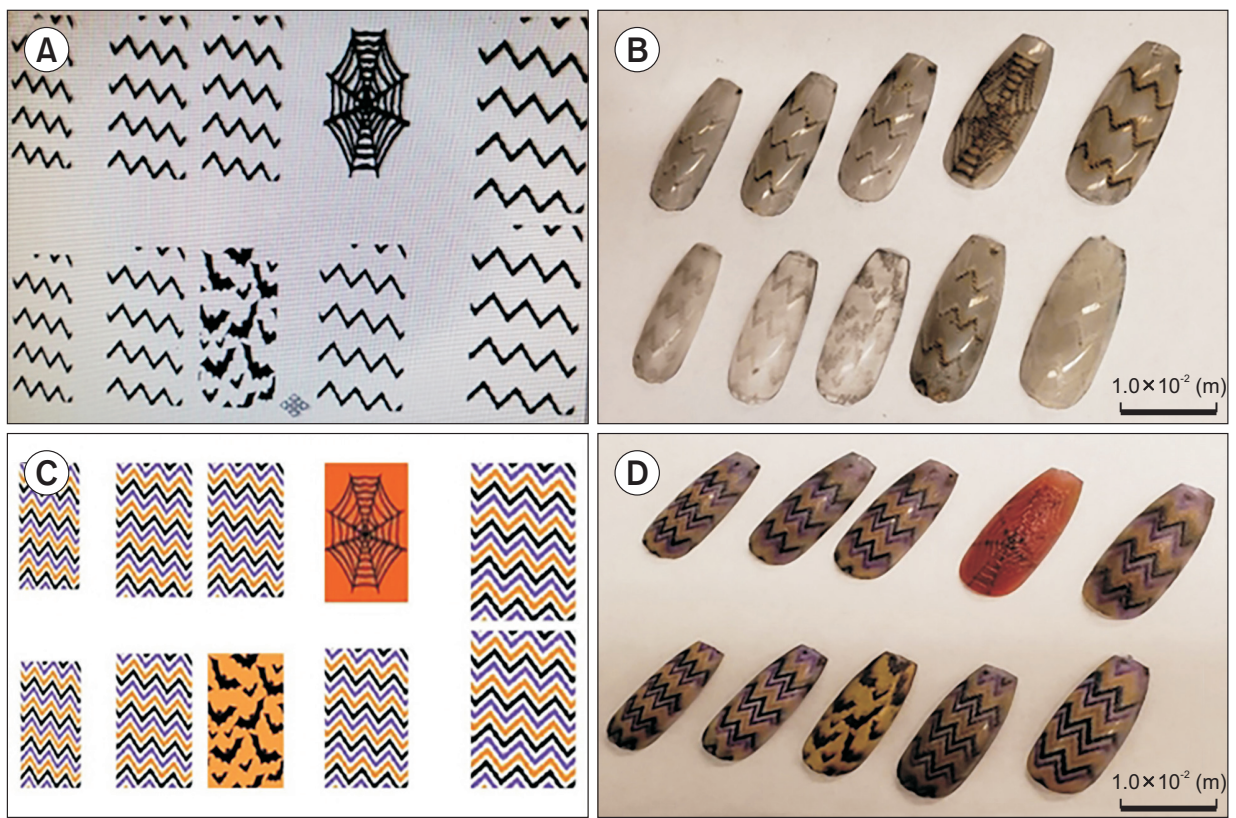

Fig. 4. Designs achieved with $2.5-\mathrm{W}$ laser-guided treatment for high definition printing on conformal surfaces: (A) nail tattoo designs before laser guide treatment; (B) nail tattoo designs after laser guide treatment; $(\mathrm{C})$ colored nail art designs before laser guide treatment; (D) colored nail art designs after laser guide treatment.

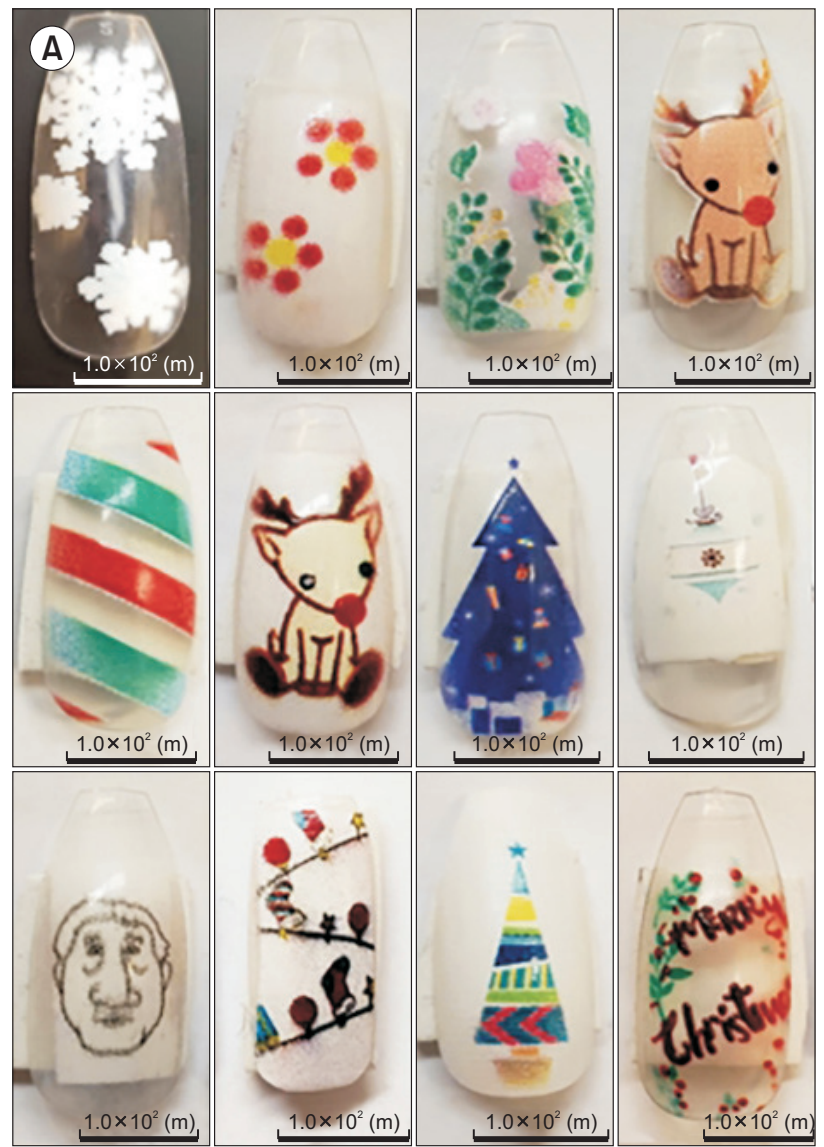

B

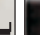

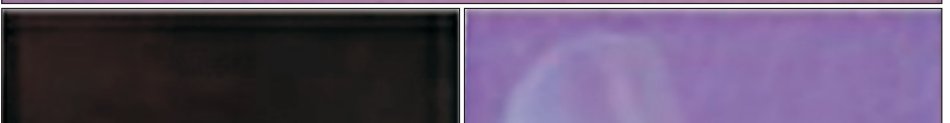

Fig. 5. Two different methods for constructing three-dimensional (3D) structures on curved nail tips: (A) repeated ink-jetting method; (B) z-axis control 3D printing method. 
$\mathrm{z}$-axis $50 \mu \mathrm{m}$ at a time, based on the application of the $\mathrm{z}$-axis changing program shown in the schematic diagram in Fig. 1. We successfully demonstrated that the repeated ink-jetting and $\mathrm{z}$-axis control printing methods were both feasible for printing 3D shapes. Generally, the total printing time did not exceed 5 minutes for the repeated ink-jetting method; however, the zaxis control printing method was problematic in that printing could consume anywhere from a minimum of 30 minutes to a maximum of 2 hours. Actual commercialization and client preferences based on the quality of the finished product were expected to vary over time.

\section{Three-dimensional nail art printing equipment implementation}

The technology for 3D printing, which has spread across society based on the adoption of fused deposition modeling (FDM), has not yet been actively applied in the nail art industry due to the sophisticated shapes and various colors required for the final products in nail art, economical 2D printing devices that implement them, and lack of material development. FDM equipment is advantageous from an economic standpoint in that it uses low-cost materials, and it is feasible for construction of 3D shapes relatively quickly, as demonstrated through simulation tests that maximized the filling rate for high viscosity materials. However, despite these advantages, the entry of printing by means of FDM into the extravagant nail art industry is considered nearly impossible because of the relatively large (over $100 \mu \mathrm{m}$ ) nozzle size currently used. Additionally, multicolor expression is more difficult to convey than a single color, and adhesiveness is severely problematic when attaching nail art directly to a fingernail surface using thermosetting resin [3]. The original technology of solidifying polymer materials via a UV lamp is owned by a 3D printer manufacturer, Objet Geometries (Tel Aviv, Rehovot, Israel), and it offers outstanding benefits in material strength and precision during the UV hardening process [4]. Objet Geometries possesses core technology for enclosing and printing its patented PolyJet polymers (Objet Geometries), and after being acquired by Stratasys, Ltd. (Los Angeles, CA, USA), a leading 3D printer manufacturer in the United States, in November 2012, it has made great progress. One of its accomplishments was expanding the current polymer 3D printing devices in the automobile, aviation, and medical industries while maintaining a high level of mechanical strength. Despite the achievements, however, there are still drawbacks. For instance, the UV lamp technology that uses mercury requires 30 minutes for preheating before the com- mencement of the process, and if it is used in a confined indoor space with a power consumption of approximately $3 \mathrm{~W}$, the indoor temperature can increase. Despite studies conducted to optimize the hardening process while reducing the heat released by using carbon nanotubes, the entry into the nail art industry, which requires the production of numerous colors in a short timeframe, could not be accomplished due to the difficulties in commercialization [5]. The stereolithography apparatus (SLA) method was developed in 1984 to address the aforementioned problems. The SLA method is ten times faster than the conventional method, and its light source evolved from a conventional laser to UV and visible light to attain sophisticated shapes. However, it is just one of many potential solutions in the nail art industry, in which complex shapes must be printed quickly and be competitively priced [6]. The typical SLA technology immerses the material into a water tank or employs a single-use process only. Nevertheless, this becomes an environmental problem, and the quantity of unnecessary compounds contained in a water tank is too excessive in comparison with the final price of the 3D material to justify its use. Furthermore, without standardization of the SLA technology method, the optimization of the process and materials remains difficult to achieve [7]. Later, a roll-to-roll method that processes UV material between rollers was attempted. However, despite it being the most suitable printing method in the nail art industry at the time, in that accurate shapes could be fabricated quickly, it had a serious deficit in that it was incapable of printing more than a single color at a time [8]. The driving force behind the growth in traditional nail art to its current level was not due to technological progress. In traditional nail art, when an error was found in a final product, it could be immediately corrected based on the experience of the nail technician, and a conversation could take place between the client and technician to remedy the problem. Therefore, emotional satisfaction due to interpersonal contact, which cannot be quantified, drove the growth of the industry to a significant level despite its low level of precision and reproducibility, unhygienic qualities, exposure to risks, and increased length of time to achieve [9]. According to Carbon 3D, Inc., a 3D printer manufacturer, the STL method did not infiltrate the nail industry because its application and target industry has changed to transparent agents for orthodontic treatment, due to its issues with colors and the cost of materials [10]. As the closeto-commercialization level of IoT-controllable devices and AI have been combined for their spatial and technical benefits, they are expected to be applied to the 3D nail art industry after 2019 [11]. The consumer-directed production technology that 
leads a fourth industry, (i.e., a small quantity batch production technology using 3D printing equipment) will be applied to nail art. Furthermore, it will simultaneously apply 3D G-code transfer technology, in which various materials such as food, metals, polymers, and compounds are controlled by the Arduino system (Arduino, Turin, Italy) and information processing hardware, such as RPI. Particularly, in the nail art realm of this study, production and consumption are expected to grow into a convergent art area of advertisement, images, and technologies through IoT and AI. For the majority of the technologies using these two technologies, the price of equipment has been the biggest stumbling block for commercialization; however, commercialization is expected to be feasible in the near future because great economic progress has been made by focusing on specific measures such as device simplification, skipping, and function reduction. Such printing technology has continually advanced due to the optimization of such factors as material spraying method, distance, solidification, and angle of light, and has shown trends of improvement in precision, size reduction, and decreased processing time [6].

Since 2015, the Korea Printed Electronics Research Association (KoPERA), led by the South Korean government, has been developing inexpensive equipment, various color expressions, easily-accessible software programs, and devices that take into consideration the risks posed by harmful chemical substances of conventional nail art in an effort to successfully enter the nail art industry. It has been reported that source technology using UV-LED instead of a UV lamp for the materials hardening process requires a mere $3 \mathrm{~W}$ (i.e., 1/10th the energy consumption of a conventional UV lamp) [12]. Furthermore, among the many technologies capable of 3D printing, the manufacturing technology that facilitated the direct printing of a unique protruding object on a nail tip was first introduced in 2007 with the advantage and capability of directly printing an environment-friendly material without using gel; however, much research regarding precision is necessary prior to being accepted by the nail art industry [13]. In 2017, the United States led the development of the first technology that transmitted the desired shape of a consumer through IoT technology from a remote area to a printing area, which incorporated safety technology and the prevention of environmental contamination, while conducting 3D printing on a curved fingernail surface and monitoring the results $[14,15]$.

\section{Three-dimensional nail art printing materials}

Gels have commonly been used in nail art as a way to control the viscosity of the materials, including oligomer, an ac- tive group compound having a double bond functional group, photoinitiator, dispersant, and dye. More specifically, it has been reported that manicures or gel nails contain chemical substances harmful to the human body, such as formaldehyde, formaldehyde resin, paraben, toluene, xylene, dibutyl phthalate, polyvinyl chloride, camphor, ethyl tosylamide, triphenyl phosphate, and acetone [16]. Because gels have stronger adhesive strength than ordinary manicures, their lasting time is increased, but they have a drawback in that they seriously damage the surface of the nail due to the lifting between the gel nail and the nail surface caused by continuous shocks. There is another problem in that UV exposure takes a long time to harden the material thickened by increased viscosity of the gel. If the inside of the material is not completely hardened due to uneven thickness, harmful carcinogens (including photoinitiator) may penetrate the epidermis structure of the nail and enter the human body, theoretically causing a more critical problem, such as skin cancer [17]. These problems require continuous research and improvement because a large amount of melanoma-inducing chemicals are contained not only in the gels but also in the adhesives used to bind artificial tips to fingernails [18]. Studies have been actively conducted to print various materials directly onto the surfaces of nails without a gel component. Furthermore, 3D shape fabrication technologies that incorporate copper metal nanoparticles [19] and ceramic materials [20] are considered environment-friendly source technologies of future printing without gel by optimizing the conditions of the printing equipment and viscosity of the material. From the precision standpoint, however, their results are not on a par with repeated ink-jetting, which sprays the material directly from a nozzle. More specifically, it has been reported that if printing is performed using a new method that increases the filling density of a material and a method of preparing such a material under high pressure, a 3D structure can be materialized quickly using a material of high viscosity; this is also effective for nanomaterials [21] and food materials [22], which show substantial changes over time. However, this technology has a problem in that the size of the nozzle tip is too large to precisely fabricate a $3 \mathrm{D}$ shape on a nail. This study determined the condition necessary to perfect the hardening process by accurately controlling the thickness of the photoinitiator to perform external hardening after printing. Such chemical substances have strong toxicity and can have harmful effects on the human body, depending on their concentrations. Underlying harmful effects still exist in the process of creating nail art. Because solutions to address the harmful chemicals contained in nail gels have been ineffec- 
tive, consumers have been increasingly raising concerns while becoming more sensitive to this issue. Therefore, the UV-LED printer proposed in this study was used to facilitate safe printing whereby consumers remained in a remote location far from potential contaminants, controlling the use of chemical substances from a long distance. This study aimed to demonstrate the feasibility of successful commercialization in the nail art industry.

\section{Internet of things three-dimensional printing and material supply}

Conventional nail art education has been time-oriented rather than result-oriented. Nail art education in the USA, Japan, and South Korea consists of hygiene and practical training and is conducted according to government policies based on the approval of private-sector-led non-profit associations. School-oriented basic education should be enhanced, and the quality of practical training in the field should be improved by shifting from time-oriented training to result-oriented training. If $3 \mathrm{D}$ printing technology is enhanced, it is expected that education programs for 3D nail art printing specialists and in-thefield education programs will substantially change to provide higher-level education, including basic physics, chemistry, and medical science, in addition to art and creativity. The apprenticeship education method of completing a given time in the field in an actual nail shop causes a large discrepancy in the quality of education among nail technicians. Because of this, nail art is considered an art, not a science. Consequently, the quality of the results is determined by the skill level of the nail technician [23]. This study would like to leave the realm of art to the consumers and instead transform nail art into a science, incorporating safer, more realistic, and reproducible standardized processes. Accordingly, this study adopted a drug delivery system that already controls 3D printing seamlessly in the pharmaceutical industry, and we have attempted to integrate it into the fabrication of nail art. This system applies the new concept of using the IoT to control the materials supply chain, whereby education is achieved by dividing the processes of safety, manufacture, and delivery, incorporating 3D printing and minimizing errors in the field [24]. In other words, this study attempted a digital fabrication method where the $3 \mathrm{D}$ nail art is determined by consumers in the field. This custom-design method has an advantage in that any shape can easily be designed and printed simply by a consumer selecting a size and selecting or designing a picture.

In conclusion, this study incorporated a design recommen- dation and printing process that was repeatedly performed using an IoT system and AI technology through Wi-Fi with 3D printing equipment. More than 1,000 designs were transmitted through the cloud to produce 3D shapes. Then, a method was attempted to turn the equipment on and off without preheating using UV LED technology. As a result, the economic feasibility, reproducibility, and safety of 3D printing were confirmed. This study established a materials supply chain, which manages the whole process by combining a cloud-connected IoT system with a nail art printer, whereby a provider prints a consumer's desired shape or pattern in an environment-friendly way from afar. Consequently, uncertain variables caused by differences in time, money, health, and skill levels of individuals inherent in the traditional handcrafted method of nail art were minimized and did not decrease either the artistic value or the level of satisfaction. Nonpermanent tattoo products were provided instead of a permanent tattoo process so that consumers could easily order sophisticated nail art and unique tattoo designs without risking exposure in a confined indoor space. In the future, it is expected that this new approach will be provided for economic feasibility, satisfaction, beauty treatment, cosmetics, and nonsurgical applications.

\section{Conflicts of interest}

The authors have nothing to disclose.

\section{References}

1. Bollard SM, Beecher SM, Moriarty N, Kelly JL, Regan PJ, Potter SM. Skin cancer risk and the use of UV nail lamps. Australas J Dermatol 2018;59:348-9.

2. Mia de GRAAF. Can gel manicures give you skin cancer? Top dermatologists explain the risks of UV exposure - and how to protect yourself [Internet]. London: Associated Newspapers Ltd.; c2019 [cited 2019 May 17]. Available from: https://www. dailymail.co.uk/health/article-7042859/Can-gel-manicuresskin-cancer-dermatologist-explains.html.

3. Amert AK, Oh DH, Kim NS. A simulation and experimental study on packing of nanoinks to attain better conductivity. J Appl Phys 2010;108:102806.

4. Jonai K, Yoshihiro Y, Nishida D, inventors; Toyo Ink SC Holdings Co Ltd., assignee. Actinic radiation curable jet-printing ink. United States patent US 8,105,679. 2012 Jan 31.

5. Ortega A, Park B, Kim NS. Printability and electrical conductivity of UV curable MWCNT ink. J Electron Mater 
2015;44:778-83.

6. Ishizaka M. inventor; Jit Ceremony Co Ltd., assignee. Nail art method and device. United States patent US 6,336,694. 2002 Jan 08.

7. Hoffman J, Hwang S, Ortega A, Kim NS, Moon K. The standardization of printable materials and direct writing systems. J Electron Packag 2013;135:011006.

8. Mendoza KL, Ortega A, Kim NS. Optimization of UV LEDcurable ink for reverse-offset roll-to-plate (RO-R2P) printing. J Electron Mater 2015;44:784-91.

9. Cooper N. The rising demand for nail art [Internet]. Conwy: NCC Resources Ltd.; c2017 [cited 2017 Oct 27]. Available from: https://www.ncchomelearning.co.uk/blog/the-risingdemand-for-nail-art/.

10. Carbon. Major orthodontics laboratory trusts Carbon's dental solution for thermoforming applications [Internet]. Redwood City (CA): Carbon; c2019 [cited 2019 Dec 1]. Available from: https://www.carbon3d.com/case-studies/majororthodontics-laboratory-trusts-carbons-dental-solution-forthermoforming-applications/2010.

11. Kim NS, Kim JH. Opportunities and challenges of 3D printing technology in beauty nail art. Paper presented at: The Korean Society of Cosmetology; 2019 Jun 8; Daejeon.

12. Kim N, Hwang S, Hong S, Hwang J, inventors; Biomedical 3D printing co Ltd., assignee. Curing apparatus for 3D printer, using ultraviolet light emitting diode. Korea patent KR101538326B1. 2013 Jun 17.

13. Kim NS, Han KN, Church KH. Direct writing technology for 21st century industries -focus on micro-dispensing deposition write technology. Transactions Korean Soc Mach Tool Eng 2007;511-5.

14. Quach T, Nguyen KD, Doan-Billings PA, Okahara L, Fan C, Reynolds P. A preliminary survey of Vietnamese nail salon workers in Alameda County, California. J Community Health
2008;33:336-43.

15. Kim NS, inventor; Biomedical 3D printing Co Ltd., assignee. Artificial nail and production equipment employing 3D printing technique for UV LED curing. Korea patent KR101898602B1. 2018 March 08.

16. Sainio EL, Engström K, Henriks-Eckerman ML, Kanerva L. Allergenic ingredients in nail polishes. Contact Dermatitis 1997;37:155-62.

17. Clark EA, Alexander MR, Irvine DJ, Roberts CJ, Wallace MJ, Sharpe S, et al. 3D printing of tablets using inkjet with UV photoinitiation. Int J Pharm 2017;529:523-30.

18. Choi H. Nails black lines, I thought, a simple injury ... 'cutaneous cancer' [Internet]. Seoul: dongA.com; c2019 [cited 2019 May 5]. Available from: http://soda.donga.com/Main/3/ all/37/1719245/1.

19. Hong S, Kim N. Synthesis of 3D printable Cu-Ag Core-Shell materials: kinetics of $\mathrm{CuO}$ film removal. J Electron Mater 2015;44:823-30.

20. Kim NP, Cho D, Zielewski M. Optimization of 3D printing parameters of Screw Type Extrusion (STE) for ceramics using the Taguchi method. Ceram Int 2019;45:2351-60.

21. Han KN, Kim NS. Challenges and opportunities in direct write technology using nano-metal particles. KONA Powder Particle J 2009;27:73-83.

22. Kim NP, Eo JS, Cho D. Optimization of piston type extrusion (PTE) techniques for 3D printed food. J Food Eng 2018;235:41-9.

23. Pan CJ, Chen MH, inventors; Helio Optoelectronics Corp., assignee. Low-light-emitting-angle high-luminance uv led nail lamp structure and led light source module thereof. United States patent US 13,343,844. 2012 Jan 05

24. Kim N, Eo J, Cepeda B, Kim J. A new paradigm of pharmaceutical drug delivery systems (DDS): challenges for space, time, and shapes. Innov Pharm 2018;9:11-24. 